\title{
ON A RESULT OF DARBOUX
}

\section{JAVIER CHAVARRIGA, JAUME LLIBRE AND JEAN MOULIN OLLAGNIER}

\begin{abstract}
This paper is concerned with a relation of Darboux in enumerative geometry, which has very useful applications in the study of polynomial vector fields. The original statement of Darboux was not correct. The present paper gives two different elementary proofs of this relation. The first one follows the ideas of Darboux, and uses basic facts about the intersection index of two plane algebraic curves; the second proof is rather more sophisticated, and gives a stronger result, which should also be very useful. The power of the relation of Darboux is then illustrated by the provision of new, simple proofs of two known results. First, it is shown that an irreducible invariant algebraic curve of degree $n>1$ without multiple points for a polynomial vector field of degree $m$ satisfies $n \leqslant m+1$. Second, a proof is given that quadratic polynomial vector fields have no algebraic limit cycles of degree 3 .
\end{abstract}

\section{Introduction}

Darboux was the first to give the following relation in enumerative geometry [2, pp. 83-84]:

On peut rattacher cette recherche à un lemme relatif à six polynômes $A, A^{\prime}, B$, $B^{\prime}, C, C^{\prime}$, de degrés $l, l^{\prime}, m, m^{\prime}, n, n^{\prime}$ satisfaisant à l'identité déjà considérée (48) $\quad A A^{\prime}+B B^{\prime}+C C^{\prime}=0$;

il est évident que les degrés des produits $A A^{\prime}, B B^{\prime}, C C^{\prime}$ sont égaux.

On a donc déjà

$$
l+l^{\prime}=m+m^{\prime}=n+n^{\prime}=\lambda .
$$

Cela posé, je dis que la somme du nombre des points communs aux trois courbes

$$
A=0, \quad B=0, \quad C=0,
$$

et du nombre des points communs aux trois courbes

$$
A^{\prime}=0, \quad B^{\prime}=0, \quad C^{\prime}=0,
$$

est égale à

$$
\frac{l m n+l^{\prime} m^{\prime} n^{\prime}}{\lambda}
$$

We shall refer to this result as the Darboux lemma; it can be stated more precisely as follows. 
Darboux lemma. Let $\mathbb{K}$ be an algebraically closed field, and let $A, B, C, A^{\prime}, B^{\prime}$ and $C^{\prime}$ be six homogeneous polynomials of degrees $l, m, n, l^{\prime}, m^{\prime}$ and $n^{\prime}$ in three variables with coefficients in $\mathbb{K}$ such that:

(i) $A, B$ and $C$ are relatively prime, and so are $A^{\prime}, B^{\prime}$ and $C^{\prime}$;

(ii) $l+l^{\prime}=m+m^{\prime}=n+n^{\prime}=r$, and the following orthogonality relation holds:

$$
A A^{\prime}+B B^{\prime}+C C^{\prime}=0
$$

(iii) the homogeneous ideal $\left(A, B, C, A^{\prime}, B^{\prime}, C^{\prime}\right)$ generated by all six polynomials has no zero in the projective plane $\mathbb{P}_{2}(\mathbb{K})$.

Then the homogeneous ideals generated by the triples $(A, B, C)$ and $\left(A^{\prime}, B^{\prime}, C^{\prime}\right)$ have only finitely many zeroes in the projective plane.

Denoting by $h$ and $h^{\prime}$ the total multiplicities $I(A, B, C)$ and $I\left(A^{\prime}, B^{\prime}, C^{\prime}\right)$ of these homogeneous ideals in the projective plane, there is a relation between $h, h^{\prime}$ and the degrees:

$$
h+h^{\prime}=\frac{l m n+l^{\prime} m^{\prime} n^{\prime}}{r}=r^{2}-r(l+m+n)+(l m+m n+n l) .
$$

\section{Darboux started the proof of his result as follows.}

En effet, soient $h$ le nombre des points communs aux trois courbes $A, B, C$; $h^{\prime}$ celui des points communs aux trois courbes $A^{\prime}, B^{\prime}, C^{\prime}$.

This original proof is wrong; in particular, Darboux paid little attention to the last hypothesis (no common zeroes), and a counterexample is easy to find. Jouanolou noticed that Darboux's result was wrong, and clearly established the formula (2) in his book [4, pp. 183-184], but his proof is far from being elementary. Jouanolou uses Chern's classes; for such a seemingly simple result, it is natural to expect a proof that relies on the use of a simpler technology.

The purpose of this paper is to give two different elementary proofs of the Darboux lemma, and to propose some applications of this result to the study of polynomial planar vector fields.

In the first proof, we use standard facts about the intersection index of plane algebraic curves. This proof is divided into two steps. The first step deals with a special case of the statement under extra assumptions, and it follows the ideas of Darboux. The second step consists in reducing the general case to the special case, in order to get the complete result.

Our second proof relies on the elementary use of exact sequences to compute the dimension of some finite-dimensional vector spaces; we receive a stronger result which could also be very useful in various applications: an inequality that holds even if the six polynomials have common projective zeroes. This proof cannot be considered as an elementary version of Jouanolou's proof; our decisive remark consists in considering the triple $\left(A^{\prime}, B^{\prime}, C^{\prime}\right)$ not only as a relation between $A, B$ and $C$, but also as a way to build an interesting ideal $\ell$ of the polynomial ring from the module of relations between $A, B$ and $C$.

The present paper is written in a self-contained way, and can thus be read independently of the given references; it is organized as follows. In Section 2 we present the results related to intersection indices that we shall need later on. We give the first proof of Darboux lemma in Section 3, correcting the proof of Darboux. A global projective proof of the Darboux lemma and an inequality are provided in Section 4. Finally, two applications of the Darboux lemma are given in Section 5.

Throughout the paper, $\mathbb{K}$ will denote an algebraically closed field. It will be convenient to denote an ideal generated in some polynomial ring over $\mathbb{K}$ by the elements $A_{1}, \cdots, A_{k}$ simply by $\left(A_{1}, \cdots, A_{k}\right)$. Other notations and definitions will be given as they are needed. 


\section{Intersection indices}

We first recall some standard facts about homogeneous ideals of the polynomial ring $\mathbb{A}=\mathbb{K}\left[x_{0}, \ldots, x_{t}\right]$ that have only a finite number of zeroes in the projective space $\mathbb{P}_{t}(\mathbb{K})$.

Let $P$ be a point in the projective space $\mathbb{P}_{t}(\mathbb{K})$. The local ring $\mathcal{O}_{P}$ can be defined in two ways.

First, it is the subring of the field $\mathbb{K}\left(x_{0}, \cdots, x_{t}\right)_{0}$ of homogeneous rational fractions of degree 0 consisting of all those with a denominator that does not vanish at $P$.

On the other hand, one of the projective coordinates of $P$ does not vanish, and there is no restriction in supposing that $x_{0}(P) \neq 0$, to fix matters. Then the polynomial ring $\mathbb{K}\left[x_{1}, \cdots, x_{t}\right]$ is isomorphic to the quotient ring of $\mathbb{K}\left[x_{0}, \cdots, x_{t}\right]$ by its ideal generated by $x_{0}-1$, and $\mathcal{O}_{P}$ is the local ring $S^{-1} \mathbb{K}\left[x_{1}, \cdots, x_{t}\right]$, where $S$ is the multiplicative set of all $t$-variable polynomials that do not vanish at $P$.

Let $P$ be a point of $\mathbb{P}_{t}(\mathbb{K})$, and let $\ell$ be a homogeneous ideal of $\mathbb{A}$. The ideal $\ell_{P}$ is the ideal of the local ring $\mathcal{O}_{P}$ generated by $\ell$. If the quotient ring $\mathcal{O}_{P} / \ell_{P}$ is a finite-dimensional vector space over $\mathbb{K}$, its dimension is called the multiplicity or the intersection index of $\ell$ at $P$; here, $I_{P}(\ell)$ is a convenient notation for this number. In particular, $I_{P}(\ell) \neq 0$ means that $P$ is a zero of $\ell$. Thus, if $\ell$ is a homogeneous ideal with a finite number of zeroes in $\mathbb{P}_{t}(\mathbb{K})$, the sum $I(\ell)=\sum_{P} I_{P}(\ell)$ over all zeroes of $\ell$ is well defined. It is called the total multiplicity, or the total intersection index or the degree of $\ell$.

Proposition 1. Let $\ell$ be a homogeneous ideal in the polynomial ring $\mathbb{A}=\mathbb{K}\left[x_{0}, \ldots, x_{t}\right]$, and suppose that $\ell$ has a finite number of zeroes in $\mathbb{P}_{t}(\mathbb{K})$. Homogeneous components $(\mathbb{A} / \ell)_{t}$ of the quotient ring $\mathbb{A} / \ell$ are finite-dimensional vector spaces over $\mathbb{K}$ that have the same dimension for $t$ large enough. This common dimension is equal to $I(\ell)$.

Proof. There is no restriction in supposing that there is no zero on the line $x_{0}=0$. All zeroes of $\ell$ then lie in the affine space in which $x_{0}$ can be chosen equal to 1 .

Consider the quotient map $\epsilon_{1}$ from $\mathbb{K}\left[x_{0}, \cdots, x_{t}\right]$ to $\mathbb{K}\left[x_{0}, \cdots, x_{t}\right] /\left(x_{0}-1\right)$ (evaluation at $\left.x_{0}=1\right)$. Its image $\epsilon_{1}\left(\mathbb{K}\left[x_{0}, \cdots, x_{t}\right]\right)$ is isomorphic to $\mathbb{K}\left[x_{1}, \cdots, x_{t}\right]$, and $\ell_{1}=\epsilon_{1}(\ell)$ is an ideal of $\mathbb{K}\left[x_{1}, \cdots, x_{t}\right]$ under this isomorphism.

The quotient ring $\mathbb{K}\left[x_{1}, \cdots, x_{t}\right] / \ell_{1}$ is a finite-dimensional vector space over $\mathbb{K}$, whose dimension is exactly the total multiplicity of $\ell$. A proof of this result can be found in the book by Fulton [3].

On the other hand, $\mathbb{K}\left[x_{1}, \cdots, x_{t}\right]$ is isomorphic to the quotient ring of $\mathbb{K}\left[x_{0}, \cdots, x_{t}\right]$ by its ideal $\left(x_{0}\right)$. Let $\ell_{0}$ denote the image of $\ell$ under this isomorphism. $\ell_{0}$ is a homogeneous ideal of $\mathbb{K}\left[x_{1}, \cdots, x_{t}\right]$, and it has no projective zero in $\mathbb{P}_{t-1}(\mathbb{K})$.

According to the projective Nullstellensatz, there exists a degree $t_{0}$ such that all homogeneous polynomials of degree at least $t_{0}$ of $\mathbb{K}\left[x_{1}, \cdots, x_{t}\right]$ are in $\ell_{0}$. In other words, every homogeneous element of degree $t \geqslant t_{0}$ in $\mathbb{A}$ can be written as the sum of an element of $\boldsymbol{l}$ and a multiple of $x_{0}$, which means that the multiplication by $x_{0}$ from $(\mathbb{A} / \ell)_{t}$ to $(\mathbb{A} / \ell)_{t+1}$ is surjective for $t \geqslant t_{0}-1$.

Thus, the sequence of dimensions decreases for $t \geqslant t_{0}-1$, and must eventually become constant; that is, there exists a $t_{1}$ such that the multiplication by $x_{0}$ from $(\mathbb{A} / \ell)_{t}$ to $(\mathbb{A} / \ell)_{t+1}$ is bijective for $t \geqslant t_{1}$.

It is now easy to check that the quotient map from $(\mathbb{A} / \ell)$ to $\mathbb{K}\left[x_{1}, \cdots, x_{t}\right] / \ell_{1}$, restricted to $(\mathbb{A} / \ell)_{t}$, is a bijection for $t \geqslant t_{1}$. 
In the case of two or more three-variable homogeneous polynomials over $\mathbb{K}, A_{1}, \cdots, A_{k}$, the intersection index $I_{P}\left(A_{1}, \cdots, A_{k}\right)$ at some point $P$ of $\mathbb{P}_{2}(\mathbb{K})$ can be defined as the corresponding index for the homogeneous ideal generated by $A_{1}, \cdots, A_{k}$, when this index is finite. We then note that $I_{P}\left(A_{1}, \cdots, A_{k}\right)=I_{P}\left(A_{1}, \cdots, A_{k}\right)$.

In particular, if $A_{1}, \cdots, A_{k}$ (with $k \geqslant 2$ ) are relatively prime, $I_{P}\left(A_{1}, \cdots, A_{k}\right)$ is defined at every point $P$. On the other hand, if they have a non-trivial greatest common divisor $D$, $I_{P}\left(A_{1}, \cdots, A_{k}\right)$ is defined at all points $P$ of $\mathbb{P}_{2}(\mathbb{K})$ where $D(P) \neq 0$.

Here are some standard properties of the intersection index whose proof can be found in Fulton's book [3]. The first two are general.

(i) $I_{P}\left(A_{1}, \cdots, A_{k}\right)$ depends only on the ideal $\left(A_{1}, \cdots, A_{k}\right)$ of $\mathbb{A}$.

(ii) In fact, $I_{P}\left(A_{1}, \cdots, A_{k}\right)$ depends only on the ideal generated by $A_{1}, \cdots, A_{k}$ in $\mathcal{O}_{P}$ : if $B(P) \neq 0$, then $B$ is invertible in $\mathcal{O}_{P}$ and $I_{P}\left(B A_{1}, A_{2}, \cdots, A_{k}\right)=I_{P}\left(A_{1}, A_{2}, \cdots, A_{k}\right)$.

The next two properties are specific to the three-variable case.

(iii) If $B$ has no non-trivial common factor with $C C^{\prime}$, then $I_{P}\left(B, C C^{\prime}\right)=I_{P}(B, C)+$ $I_{P}\left(B, C^{\prime}\right)$ (addition formula).

(iv) If $F$ and $G$ are two homogeneous polynomials without a non-trivial common factor, they have a finite number of common projective zeroes and $I(F, G)=\operatorname{deg}(F) \cdot \operatorname{deg}(G)$ (Bézout's theorem).

\section{Correcting the proof given by Darboux}

Using standard properties of the intersection index, we propose now a correct proof of the Darboux lemma. Let us begin with some definitions and notations.

We shall call a family $\left[A, B, C, A^{\prime}, B^{\prime}, C^{\prime}\right]$ of homogeneous polynomials in $\mathbb{K}[x, y, z]$ an orthogonal system of polynomials if

(i) $A, B$ and $C$ are relatively prime, and so are $A^{\prime}, B^{\prime}$ and $C^{\prime}$;

(ii) $\operatorname{deg}(A)+\operatorname{deg}\left(A^{\prime}\right)=\operatorname{deg}(B)+\operatorname{deg}\left(B^{\prime}\right)=\operatorname{deg}(C)+\operatorname{deg}\left(C^{\prime}\right)=\rho\left(A, B, C, A^{\prime}, B^{\prime}, C^{\prime}\right)$, in which case $\rho\left(A, B, C, A^{\prime}, B^{\prime}, C^{\prime}\right)$ is called the degree of the system,

(iii) the orthogonality condition (1), that $A A^{\prime}+B B^{\prime}+C C^{\prime}=0$, holds.

We shall say that an orthogonal system of polynomials $\left[A, B, C, A^{\prime}, B^{\prime}, C^{\prime}\right]$ is without projective zero if $A, B, C, A^{\prime}, B^{\prime}$ and $C^{\prime}$ have no common zero in the projective plane.

If $\left[A, B, C, A^{\prime}, B^{\prime}, C^{\prime}\right]$ is an orthogonal system of polynomials, then $A, B$ and $C$ are relatively prime, the total intersection index $I(A, B, C)$ is well-defined, and so is $I\left(A^{\prime}, B^{\prime}, C^{\prime}\right)$.

Now denote the degrees of the polynomials $A, B, C, A^{\prime}, B^{\prime}$ and $C^{\prime}$ by $l, m, n, l^{\prime}, m^{\prime}$ and $n^{\prime}$, respectively, and the degree of the orthogonal system $\rho\left(A, B, C, A^{\prime}, B^{\prime}, C^{\prime}\right)$ by $r$, to simplify the discussion.

The ratio

$$
\frac{l m n+l^{\prime} m^{\prime} n^{\prime}}{r}=r^{2}-r(l+m+n)+(l m+m n+n l)
$$

is a well-defined positive integer, which is 0 when $r=0$. We then denote

$$
\begin{aligned}
\Delta\left(A, B, C, A^{\prime}, B^{\prime}, C^{\prime}\right) & =I(A, B, C)+I\left(A^{\prime}, B^{\prime}, C^{\prime}\right)-\frac{l m n+l^{\prime} m^{\prime} n^{\prime}}{r} \\
& =h+h^{\prime}-\frac{l m n+l^{\prime} m^{\prime} n^{\prime}}{r},
\end{aligned}
$$

and we call this difference the gap of the orthogonal system. 
With these definitions, the Darboux lemma can be stated as follows.

The gap is zero for an orthogonal system of polynomials without projective zero.

We first give the result under the additional assumption that all six polynomials are pairwise relatively prime, except maybe $A$ and $A^{\prime}, B$ and $B^{\prime}, C$ and $C^{\prime}$; in other words, we suppose that there is no non-trivial common factor to $A A^{\prime}, B B^{\prime}, C C^{\prime}$, and we then say that the orthogonal system is irreducible. This proof follows the ideas of Darboux. We shall reduce the general case to this special case later.

In our opinion, it is convenient and non-confusing to identify a homogeneous non-zero three-variable polynomial $F$ with the projective planar curve $F=0$ that it defines. We thus follow the free intuitive notations of Darboux.

For instance, the notation ' $P \in A \cap B$ ' means that the point $P$ of $\mathbb{P}_{2}(\mathbb{K})$ is a common zero of the two homogeneous polynomials $A$ and $B$, and belongs to the intersection of the two curves $A=0$ and $B=0$, as well as the alternative notation ' $A(P)=0$ and $B(P)=0$ '.

Proposition 2. Let $\left[A, B, C, A^{\prime}, B^{\prime}, C^{\prime}\right]$ be an irreducible orthogonal system of polynomials without projective zero. Then $\Delta\left(A, B, C, A^{\prime}, B^{\prime}, C^{\prime}\right)=0$.

Proof. We first notice that $I_{P}\left(A, B, C C^{\prime}\right)=I_{P}\left(A, B,-A A^{\prime}-B B^{\prime}\right)=I_{P}(A, B)$ at every point $P$ of $\mathbb{P}_{2}(\mathbb{K})$, according to Section 2 . We want to prove the following equality at $P \in A \cap B$ :

$$
I_{P}(A, B)=I_{P}\left(A, B, C C^{\prime}\right)=I_{P}(A, B, C)+I_{P}\left(A, B, C^{\prime}\right) .
$$

From the orthogonality relation (1), $P \in C C^{\prime}$ and, by replacing $A, B$ and $C$ by $A^{\prime}, B^{\prime}$ and $C^{\prime}$, there is no restriction in supposing that $C(P)=0$.

If $C^{\prime}(P) \neq 0$, then $I_{P}\left(A, B, C^{\prime}\right)=0$. According to Section 2, we know that

$$
I_{P}(A, B, C)=I_{P}\left(A, B, C C^{\prime}\right)=I_{P}(A, B),
$$

and equality (3) holds in this case.

We now suppose that $C^{\prime}(P)=0$. Since $A \cap A^{\prime} \cap B \cap B^{\prime} \cap C \cap C^{\prime}=\emptyset$, either $A^{\prime}(P) \neq 0$ or $B^{\prime}(P) \neq 0$, and there is no restriction in supposing that $A^{\prime}(P) \neq 0$. Then, according to Section 2,

$$
\begin{aligned}
& I_{P}(A, B, C)=I_{P}\left(A^{\prime} A, B, C\right)=I_{P}(B, C), \\
& I_{P}\left(A, B, C^{\prime}\right)=I_{P}\left(A^{\prime} A, B, C^{\prime}\right)=I_{P}\left(B, C^{\prime}\right), \\
& I_{P}\left(A, B, C C^{\prime}\right)=I_{P}\left(A^{\prime} A, B, C C^{\prime}\right)=I_{P}\left(B, C C^{\prime}\right) .
\end{aligned}
$$

As $B$ has no non-trivial common factor with $C$ or $C^{\prime}$, equality (3) follows from the addition formula of the intersection index: $I_{P}\left(B, C C^{\prime}\right)=I_{P}(B, C)+I_{P}\left(B, C^{\prime}\right)$. The use of the addition formula is the only place where the extra assumption that the orthogonal system is irreducible plays a role.

Summing the intersection indices at all points $P \in A \cap B$, relation (3) leads to

$$
\begin{aligned}
l m & =I(A, B) \\
& =\sum_{P \in A \cap B} I_{P}(A, B) \\
& =\sum_{P \in A \cap B} I_{P}\left(A, B, C C^{\prime}\right) \\
& =\sum_{P \in A \cap B} I_{P}(A, B, C)+\sum_{P \in A \cap B} I_{P}\left(A, B, C^{\prime}\right) \\
& =h+(l m-h),
\end{aligned}
$$

which means that the total intersection index $I\left(A, B, C^{\prime}\right)$ is $l m-h$. 
Similar considerations lead to the next two equalities.

The first one,

$$
\begin{aligned}
l n^{\prime} & =I\left(A, C^{\prime}\right) \\
& =\sum_{P \in A \cap C^{\prime}} I_{P}\left(A, C^{\prime}\right) \\
& =\sum_{P \in A \cap C^{\prime}} I_{P}\left(A, B B^{\prime}, C^{\prime}\right) \\
& =\sum_{P \in A \cap C^{\prime}} I_{P}\left(A, B, C^{\prime}\right)+\sum_{P \in A \cap C^{\prime}} I_{P}\left(A, B^{\prime}, C^{\prime}\right) \\
& =(l m-h)+\left(l n^{\prime}-(l n-h)\right),
\end{aligned}
$$

means that the total intersection index $I\left(A, B^{\prime}, C^{\prime}\right)$ is $l n^{\prime}-l m+h$.

The second one,

$$
\begin{aligned}
m^{\prime} n^{\prime} & =I\left(B^{\prime}, C^{\prime}\right) \\
& =\sum_{P \in B^{\prime} \cap C^{\prime}} I_{P}\left(B^{\prime}, C^{\prime}\right) \\
& =\sum_{P \in B^{\prime} \cap C^{\prime}} I_{P}\left(A A^{\prime}, B^{\prime}, C^{\prime}\right) \\
& =\sum_{P \in B^{\prime} \cap C^{\prime}} I_{P}\left(A, B^{\prime}, C^{\prime}\right)+\sum_{P \in B^{\prime} \cap C^{\prime}} I_{P}\left(A^{\prime}, B^{\prime}, C^{\prime}\right) \\
& =l n^{\prime}-l m+h+h^{\prime},
\end{aligned}
$$

means that the total intersection index $I\left(A^{\prime}, B^{\prime}, C^{\prime}\right)$ is $m^{\prime} n^{\prime}-l n^{\prime}+l m-h$. From this last result, we deduce that $\Delta\left(A, B, C, A^{\prime}, B^{\prime}, C^{\prime}\right)=0$.

In order to prove the Darboux lemma in the general case, we need a way to reduce non-irreducible orthogonal systems to irreducible ones. Let $\left[A, B, C, A^{\prime}, B^{\prime}, C^{\prime}\right]$ be a nonirreducible orthogonal system of polynomials. The orthogonality relation easily implies that two polynomials of the same triple have a non-trivial greatest common divisor (gcd), and there is no restriction in supposing that $D=\operatorname{gcd}(A, B) \notin \mathbb{K}$ to describe what a reduction is.

According to the orthogonality relation, $D$ also divides $C^{\prime}$ as it is coprime with $C$, and we have $A=D A_{1}, B=D B_{1}, C^{\prime}=D C_{1}^{\prime}$, with $\operatorname{gcd}\left(A_{1}, B_{1}\right)=1$. So $\left[A_{1}, B_{1}, C, A^{\prime}, B^{\prime}, C_{1}^{\prime}\right]$ is another orthogonal system of polynomials. If $\left[A, B, C, A^{\prime}, B^{\prime}, C^{\prime}\right]$ is without projective zero, so is $\left[A_{1}, B_{1}, C, A^{\prime}, B^{\prime}, C_{1}^{\prime}\right]$. We say that $\left[A_{1}, B_{1}, C, A^{\prime}, B^{\prime}, C_{1}^{\prime}\right]$ is a one-step reduction of $\left[A, B, C, A^{\prime}, B^{\prime}, C^{\prime}\right]$.

There are as many possible one-step reductions of an orthogonal system of polynomials as there are pairs of non-coprime polynomials of the same triple. Thus, after at most six successive one-step reductions, we get an irreducible orthogonal system that can be called the complete reduction of the original one.

The following lemma is then the key to deducing the general case of the Darboux lemma from the special case of irreducible orthogonal systems.

Lemma 3. Let $\left[A, B, C, A^{\prime}, B^{\prime}, C^{\prime}\right]$ be a non-irreducible orthogonal system of polynomials without projective zero and such that $D=\operatorname{gcd}(A, B) \notin \mathbb{K}$. If $\left[A_{1}, B_{1}, C, A^{\prime}, B^{\prime}, C_{1}^{\prime}\right]$ is the corresponding one-step reduction of $\left[A, B, C, A^{\prime}, B^{\prime}, C^{\prime}\right]$, then

$$
\Delta\left(A, B, C, A^{\prime}, B^{\prime}, C^{\prime}\right)=\Delta\left(A_{1}, B_{1}, C, A^{\prime}, B^{\prime}, C_{1}^{\prime}\right) .
$$


Proof. Let us denote by $s$ the degree of $D$, so that

$$
\begin{array}{lll}
\operatorname{deg}\left(A_{1}\right) & =l_{1}=l-s, \\
\operatorname{deg}\left(B_{1}\right) & =m_{1}=m-s, \\
\operatorname{deg}\left(C_{1}^{\prime}\right) & =n_{1}^{\prime}=n^{\prime}-s, \\
\rho\left(A_{1}, B_{1}, C, A^{\prime}, B^{\prime}, C_{1}^{\prime}\right) & =r_{1}=r-s .
\end{array}
$$

Here, $h_{1}$ will stand for $I\left(A_{1}, B_{1}, C\right)$ and $h_{1}^{\prime}$ for $I\left(A_{1}, B_{1}, C\right)$.

With these notations, after straightforward cancellations, proving the result amounts to proving that

$$
\left(h-h_{1}\right)+\left(h^{\prime}-h_{1}^{\prime}\right)=n s .
$$

This relation (8) will come from $h-h_{1}=n s$ and $h^{\prime}-h_{1}^{\prime}=0$.

We first show that $h-h_{1}=n s$ by proving the following equality for all $P \in A \cap B \cap C$ :

$$
I_{P}(A, B, C)=I_{P}\left(A_{1}, B_{1}, C\right)+I_{P}(D, C) .
$$

Let $P$ belong to $D \cap C$. Since $A \cap A^{\prime} \cap B \cap B^{\prime} \cap C \cap C^{\prime}=\emptyset$, either $A^{\prime}(P) \neq 0$ or $B^{\prime}(P) \neq 0$, and there is no restriction in supposing that $A^{\prime}(P) \neq 0$.

Let $D^{\prime}$ then be the greatest common divisor of $B$ and $C: B=D^{\prime} B_{2}, C=D^{\prime} C_{2}$. As $A, B$ and $C$ are relatively prime, $D^{\prime}$ is relatively prime with $D$, and it divides $B_{1}: B_{1}=D^{\prime} B_{3}$; it also divides $A^{\prime}$, and thus $D^{\prime}(P) \neq 0$. Then, according to Section $2, I_{P}(B, C)$ and $I_{P}\left(B_{1}, C\right)$ are well-defined and the following equalities hold:

$$
\begin{gathered}
I_{P}(A, B, C)=I_{P}\left(A A^{\prime}, B, C\right)=I_{P}(B, C), \\
I_{P}\left(A_{1}, B_{1}, C\right)=I_{P}\left(A_{1} A^{\prime}, B_{1}, C\right)=I_{P}\left(B_{1}, C\right) .
\end{gathered}
$$

Now, the addition formula of the intersection index gives

$$
I_{P}(B, C)=I_{P}\left(B_{2}, C_{2}\right)=I_{P}\left(B_{3}, C_{2}\right)+I_{P}\left(D, C_{2}\right)=I_{P}\left(B_{1}, C\right)+I_{P}(D, C) .
$$

Thus, for a $P$ in $D \cap C$, equality (9) holds.

Consider now a point $P$ in $A \cap B \cap C$ that does not belong to $D$. Then $I_{P}(D, C)=0$, whereas $I_{P}(A, B, C)=I_{P}\left(A_{1}, B_{1}, C\right)$, and equality (9) also holds.

Now, summing equality (9) over all $P \in A \cap B \cap C$ gives

$$
h=I(A, B, C)=I\left(A_{1}, B_{1}, C\right)+I(D, C)=h_{1}+s n,
$$

according to Bézout's theorem.

Now we show that $h^{\prime}-h_{1}^{\prime}=0$ by proving that $I_{P}\left(A^{\prime}, B^{\prime}, C^{\prime}\right)=I_{P}\left(A^{\prime}, B^{\prime}, C_{1}^{\prime}\right)$ at all points $P$ of $A^{\prime} \cap B^{\prime} \cap C^{\prime}$.

If $P$ belongs to $A^{\prime} \cap B^{\prime} \cap C^{\prime}$ without being in $D$, then, according to Section 2, we see that $I_{P}\left(A^{\prime}, B^{\prime}, C^{\prime}\right)=I_{P}\left(A^{\prime}, B^{\prime}, D C_{1}^{\prime}\right)=I_{P}\left(A^{\prime}, B^{\prime}, C_{1}^{\prime}\right)$.

If $P$ belongs to $A^{\prime} \cap B^{\prime} \cap D$, then $P$ does not belong to $C$, and

$$
I_{P}\left(A^{\prime}, B^{\prime}, C^{\prime}\right)=I_{P}\left(A^{\prime}, B^{\prime}, C C^{\prime}\right)=I_{P}\left(A^{\prime}, B^{\prime}\right)=I_{P}\left(A^{\prime}, B^{\prime}, C C_{1}\right)=I_{P}\left(A^{\prime}, B^{\prime}, C_{1}^{\prime}\right) .
$$

We can now draw the following conclusion.

Corollary 4 (Darboux lemma). Let $\left[A, B, C, A^{\prime}, B^{\prime}, C^{\prime}\right]$ be an orthogonal system of polynomials without projective zero. Then $\Delta\left(A, B, C, A^{\prime}, B^{\prime}, C^{\prime}\right)=0$. 
Proof. If $\left[A_{1}, B_{1}, C_{1}, A_{1}^{\prime}, B_{1}^{\prime}, C_{1}^{\prime}\right]$ is the complete reduction of $\left[A, B, C, A^{\prime}, B^{\prime}, C^{\prime}\right]$, then, according to Lemma $3, \Delta\left(A, B, C, A^{\prime}, B^{\prime}, C^{\prime}\right)$ is the same as $\Delta\left(A_{1}, B_{1}, C_{1}, A_{1}^{\prime}, B_{1}^{\prime}, C_{1}^{\prime}\right)$ and, according to Proposition $2, \Delta\left(A_{1}, B_{1}, C_{1}, A_{1}^{\prime}, B_{1}^{\prime}, C_{1}^{\prime}\right)=0$ for an irreducible orthogonal system of polynomials.

\section{A global projective proof and an inequality}

In the present section, we give another proof of the Darboux lemma. Instead of looking at all points of the intersection of several curves in the projective plane, we shall deal directly with the global multiplicity of a homogeneous ideal in $\mathbb{K}[x, y, z]$ with finitely many zeroes. Moreover, this enables us to produce a stronger result when the assumption that the orthogonal system of polynomials has no projective zeroes does not hold.

In this section, where we deal with modules over $\mathbb{K}[x, y, z]$, it is convenient to denote this polynomial ring by $\mathbb{A}$. The ring $\mathbb{A}$ is graded by the degree, its homogeneous components $\mathbb{A}_{k}$ are finite-dimensional vector spaces over $\mathbb{K}$, and their dimensions are given by $\operatorname{dim}\left(\mathbb{A}_{k}\right)=\delta(k)$, for every nonnegative integer $k$, where the integer function $\delta$ is defined by

$$
\delta(k)=\frac{(k+1)(k+2)}{2}, \quad \forall k \in \mathbb{N} .
$$

This function $\delta$ will also be useful to express the dimension of homogeneous components of $\mathbb{A}^{3}$ for a suitable graduation.

Proposition 5. Let $A, B$ and $C$ be three homogeneous polynomials without a non-trivial common factor in $\mathbb{A}$, and let $l, m$ and $n$ be their degrees. The total multiplicity $I(A, B, C)$ is equal to

$$
\delta(k)-\delta(k-l)-\delta(k-m)-\delta(k-n)+\operatorname{dim}\left(\mathrm{R}(A, B, C)_{k},\right.
$$

for every large enough integer $k$, where $\mathrm{R}(A, B, C)_{k}$ is the homogeneous component of degree $k$ of the module $\mathrm{R}(A, B, C)$ of all relations between $A, B$ and $C$. The relevant graduation of $\mathrm{R}(A, B, C)$ is induced by the graduation of the $\mathbb{A}$-module $\mathbb{A}^{3}$ in which the homogeneous component of degree $k$ is the product $\mathbb{A}_{k-l} \times \mathbb{A}_{k-m} \times \mathbb{A}_{k-n}$.

Proof. In order to begin the computation of $I(A, B, C)$, first consider the exact sequence of $\mathbb{A}$-modules:

$$
0 \stackrel{0}{\longrightarrow} \mathrm{R}(A, B, C) \stackrel{i}{\longrightarrow} \mathbb{A}^{3} \stackrel{\bullet V}{\longrightarrow} \mathbb{A} \stackrel{s}{\longrightarrow} \mathbb{A} /(A, B, C) \stackrel{0}{\longrightarrow} 0,
$$

in which

(i) the first $\mathbb{A}$-module $\mathrm{R}(A, B, C)$ is a submodule of $\mathbb{A}^{3}$, and consists of all relations $\left[r_{1}, r_{2}, r_{3}\right]$ between $A, B, C:\left[r_{1}, r_{2}, r_{3}\right] \in \mathrm{R}(A, B, C) \Leftrightarrow r_{1} A+r_{2} B+r_{3} C=0$;

(ii) the morphism $\bullet V$ from $\mathbb{A}^{3}$ to $\mathbb{A}$ is the 'scalar product by the vector $V=[A, B, C]$ of $\mathbb{K}(x, y, z)^{3}: \bullet V\left(\left[r_{1}, r_{2}, r_{3}\right]\right)=r_{1} A+r_{2} B+r_{3} C$ and $\mathrm{R}(A, B, C)$ is thus its kernel;

(iii) $s$ is the surjective quotient map from $\mathbb{A}$ to its quotient by the ideal $(A, B, C)$.

By stating that, for every nonnegative integer $k$, the homogeneous component $\left(\mathbb{A}^{3}\right)_{k}$ of degree $k$ of $\mathbb{A}^{3}$ is $\mathbb{A}_{k-l} \times \mathbb{A}_{k-m} \times \mathbb{A}_{k-n}$, we define a graduation on $\mathbb{A}^{3}$; endowed with this graduation, $\mathbb{A}^{3}$ is a graded $\mathbb{A}$-module and so is its submodule $\mathrm{R}(A, B, C)$. Moreover, all morphisms of the exact sequence (12) are homogeneous and have the degree 0 . 
Given a degree $k$, the dimension of $(\mathbb{A} /(A, B, C))_{k}$ is related to $\operatorname{dim}\left(\mathrm{R}(A, B, C)_{k}\right)$ by the alternate sum of dimensions in an exact sequence of finite-dimensional vector spaces:

$$
\operatorname{dim}\left((\mathbb{A} /(A, B, C))_{k}\right)=\operatorname{dim}\left((\mathbb{A})_{k}\right)-\operatorname{dim}\left(\left(\mathbb{A}^{3}\right)_{k}\right)+\operatorname{dim}\left(\mathrm{R}(A, B, C)_{k}\right) .
$$

If $k \geqslant \max (l, m, n)$, it is easy to see that $\operatorname{dim}\left(\left(\mathbb{A}^{3}\right)_{k}\right)=\delta(k-l)+\delta(k-m)+\delta(k-n)$, from which we deduce that

$$
\begin{aligned}
\operatorname{dim}\left((\mathbb{A} /(A, B, C))_{k}\right)= & \delta(k)-\delta(k-l)-\delta(k-m)-\delta(k-n) \\
& +\operatorname{dim}\left(\mathrm{R}(A, B, C)_{k}\right) .
\end{aligned}
$$

As $I(A, B, C)=\operatorname{dim}\left((\mathbb{A} /(A, B, C))_{k}\right)$ for a large enough $k$ according to Proposition 1 , we get the required formula for the index.

If Proposition 5 relies on the classical idea to compute the total intersection index from a consideration of the homogeneous components of the module of relations, the next proposition uses the orthogonality relation (1) between the two triples $[A, B, C]$ and $\left[A^{\prime}, B^{\prime}, C^{\prime}\right]$ to link the unknown number $\operatorname{dim}\left(\mathrm{R}(A, B, C)_{k}\right)$ to some numerical invariants related to $A^{\prime}$, $B^{\prime}$ and $C^{\prime}$.

Proposition 6. Let $\left[A, B, C, A^{\prime}, B^{\prime}, C^{\prime}\right]$ be an orthogonal system of polynomials. Then it is possible to construct an $\mathbb{A}$-linear homogeneous map from the module $\mathrm{R}(A, B, C)$ of relations between $A, B$ and $C$ to $\mathbb{A}$, whose kernel is the principal $\mathbb{A}$-module generated by the particular relation $\left[A^{\prime}, B^{\prime}, C^{\prime}\right]$, and whose image $g$ is a homogeneous ideal of $\mathbb{A}$ such that:

$$
\left(A^{\prime}, B^{\prime}, C^{\prime}\right) \subset g \subset\left(A^{\prime}, B^{\prime}, C^{\prime}\right):(A, B, C) .
$$

Moreover, the following relation holds:

$$
I(A, B, C)+I(\mathcal{g})=\frac{l m n+l^{\prime} m^{\prime} n^{\prime}}{r},
$$

where $l, m, n, l^{\prime}, m^{\prime}$ and $n^{\prime}$ are the degrees of $A, B, C, A^{\prime}, B^{\prime}$ and $C^{\prime}$, and $r$ is the degree of the orthogonal system.

Proof. First define an $\mathbb{A}$-linear homogeneous map $\wedge W$ from $\mathbb{A}^{3}$ to $\mathbb{A}^{3}$ :

$$
\wedge W\left(\left[r_{1}, r_{2}, r_{3}\right]\right)=\left[B^{\prime} r_{3}-C^{\prime} r_{2}, C^{\prime} r_{1}-A^{\prime} r_{3}, A^{\prime} r_{2}-B^{\prime} r_{1}\right]
$$

It is easy to check that if $\left[r_{1}, r_{2}, r_{3}\right] \in \mathrm{R}(A, B, C)$, then $\wedge W\left(\left[r_{1}, r_{2}, r_{3}\right]\right)$ is collinear to $V$ in $\mathbb{K}(x, y, z)^{3}$. As $V$ is irreducible, $\wedge W\left(\left[r_{1}, r_{2}, r_{3}\right]\right)$ has to be a multiple of $V$ by some polynomial $\phi\left(\left[r_{1}, r_{2}, r_{3}\right]\right)$. The desired map $\phi$ is thus defined.

As $W$ is irreducible, $\phi\left(\left[r_{1}, r_{2}, r_{3}\right]\right)=0$ if and only if $\left[r_{1}, r_{2}, r_{3}\right]$ is a multiple of $\left[A^{\prime}, B^{\prime}, C^{\prime}\right]$.

We then get a second exact sequence:

$$
0 \stackrel{0}{\longrightarrow} \mathbb{A} \stackrel{\vec{W}}{\longrightarrow} \mathrm{R}(A, B, C) \stackrel{\phi}{\longrightarrow} \mathcal{g} \stackrel{0}{\longrightarrow} 0,
$$

in which $\vec{W}$ stands for the map $P \rightarrow P\left[A^{\prime}, B^{\prime}, C^{\prime}\right]$ from $\mathbb{A}$ to $\mathbb{A}^{3}$.

The second inclusion $g \subset\left(A^{\prime}, B^{\prime}, C^{\prime}\right):(A, B, C)$ comes inherently from the definition of $\phi$ : if $\phi\left(\left[r_{1}, r_{2}, r_{3}\right]\right) \in \mathcal{g}$, the coordinates of $\phi\left(\left[r_{1}, r_{2}, r_{3}\right]\right) V=\wedge W\left(\left[r_{1}, r_{2}, r_{3}\right]\right)$ belong to $\left(A^{\prime}, B^{\prime}, C^{\prime}\right)$ and thus $\phi\left(\left[r_{1}, r_{2}, r_{3}\right]\right)$ brings $(A, B, C)$ to $\left(A^{\prime}, B^{\prime}, C^{\prime}\right)$. 
To prove the first inclusion $\left(A^{\prime}, B^{\prime}, C^{\prime}\right) \subset \mathcal{g}$, it suffices to check that $A^{\prime}, B^{\prime}$ and $C^{\prime}$ are in $\mathrm{g}$. We simply remark that $[0, C,-B]$ is a relation, and that

$$
\wedge W([0, C,-B])=\left[-B^{\prime} B-C^{\prime} C, A^{\prime} B, A^{\prime} C\right]=A^{\prime}[A, B, C],
$$

according to the orthogonality relation.

With respect to the graduation defined in Proposition 5 on $\mathbb{A}^{3}, \vec{W}$ is homogeneous of degree $r$, and $\phi$ is homogeneous of degree $r-l-m-n$; then, for any degree $k$, we deduce an exact sequence of $\mathbb{K}$-linear maps between finite-dimensional vector spaces over $\mathbb{K}$ and the corresponding formula relating the dimensions of these vector spaces:

$$
\operatorname{dim}\left(\mathrm{R}(A, B, C)_{k}\right)=\operatorname{dim}\left(\mathscr{g}_{k+r-l-m-n}\right)+\operatorname{dim}\left(\mathbb{A}_{k-r}\right) .
$$

Formula (15) now comes from identity (13), together with identity (18) and the following self-evident equality:

$$
\operatorname{dim}\left(\mathcal{g}_{k+r-l-m-n}\right)=\operatorname{dim}\left(\mathbb{A}_{k+r-l-m-n}\right)-\operatorname{dim}\left((\mathbb{A} / \mathcal{g})_{k+r-l-m-n}\right) .
$$

Adding the three equalities (13), (18) and (19), for $k$ large enough, leads to

$$
\begin{aligned}
I(A, B, C)+I(\mathcal{g})= & \delta(k)-\delta(k-l)-\delta(k-m)-\delta(k-n) \\
& +\delta(k-r)+\delta(k+r-l-m-n) \\
= & r^{2}-r(l+m+n)+l m+m n+n l .
\end{aligned}
$$

This is the required result, which does not depend on $k$.

Corollary 7. Under the hypotheses of Proposition 6 and with the notations of its proof, the following inequality holds:

$$
I(A, B, C)+I\left(A^{\prime}, B^{\prime}, C^{\prime}\right) \geqslant r^{2}-r(l+m+n)+l m+m n+n l=\frac{l m n+l^{\prime} m^{\prime} n^{\prime}}{r} .
$$

Proof. This is an easy consequence of the inclusion $\left(A^{\prime}, B^{\prime}, C^{\prime}\right) \subseteq \mathcal{g}$.

Our second corollary gives the result of Darboux; first, however, we need a lemma to complete its proof.

Lemma 8. Let $I_{1}$ and $I_{2}$ be two homogeneous ideals in $\mathbb{K}\left[x_{0}, \cdots, x_{t}\right]$ with a finite number of projective zeroes. If the homogeneous component $\left(I_{1}+I_{2}\right)_{k}$ is equal to $\mathbb{K}\left[x_{0}, \cdots, x_{t}\right]_{k}$ for $k$ large enough (we shall say that $I_{1}+I_{2}$ is full in high degree), then $I\left(I_{1}\right)=I\left(I_{1}: I_{2}\right)$.

Proof. The quotient ideal $I_{1}: I_{2}$ is equal to the quotient $I_{1}: I_{3}$, where $I_{3}=I_{1}+I_{2}$ and $I_{3}$ is full in high degree, which means that all homogeneous polynomials of degree large enough are elements of $I_{3}$.

As $I_{1}$ has a finite number of projective zeroes, there is no restriction in supposing that it has no zero on the line $x_{0}=0$. Thus, referring to the proof of Proposition 1, there exists a degree $k_{0}$ such that a homogeneous polynomial $f$ of degree $k \geqslant k_{0}$ belongs to $I_{1}$ as soon as $x_{0} f$ belongs to $I_{1}$.

As $I_{3}$ is full in high degree, for every homogeneous $f$ of degree $k \geqslant k_{0}$ in $I_{1}: I_{3}$, there exists a power $x_{0}^{d}$ of $x_{0}$ such that $x_{0}^{d} f$ belongs to $I_{1}$. Thus, $f$ itself belongs to $I_{1}$. 
Corollary 9. Under the hypotheses of Proposition 6, if moreover there is no common projective zero to all six polynomials $A, B, C, A^{\prime}, B^{\prime}$ and $C^{\prime}$, the Darboux formula holds:

$$
I(A, B, C)+I\left(A^{\prime}, B^{\prime}, C^{\prime}\right)=r^{2}-r(l+m+n)+l m+m n+n l=\frac{l m n+l^{\prime} m^{\prime} n^{\prime}}{r} .
$$

Proof. It suffices to prove that $I\left(A^{\prime}, B^{\prime}, C^{\prime}\right)=I(g)$.

From the double inclusion $\left(A^{\prime}, B^{\prime}, C^{\prime}\right) \subset \mathcal{g} \subset\left(A^{\prime}, B^{\prime}, C^{\prime}\right):(A, B, C)$, we know that

$$
I\left(A^{\prime}, B^{\prime}, C^{\prime}\right) \geqslant I(g) \geqslant I\left(\left(A^{\prime}, B^{\prime}, C^{\prime}\right):(A, B, C)\right) .
$$

The additional assumption that there is no common projective zero to all six polynomials, $A, B, C, A^{\prime}, B^{\prime}$ and $C^{\prime}$, shows that the sum of the two homogeneous ideals $(A, B, C)$ and $\left(A^{\prime}, B^{\prime}, C^{\prime}\right)$ is full in high degree; thus, according to Lemma 8 , the total multiplicities $I\left(A^{\prime}, B^{\prime}, C^{\prime}\right)$ and $I\left(\left(A^{\prime}, B^{\prime}, C^{\prime}\right):(A, B, C)\right)$ are the same.

Remark 1. In this second proof, the two triples play different roles; $[A, B, C]$ is given, and $\left[A^{\prime}, B^{\prime}, C^{\prime}\right]$ is some relation between $A, B$ and $C$, with additional properties.

It is not always true that there exists a relation for which the Darboux relation (2) holds. For instance, if $[A, B, C]$ is $\left[x^{2}, x y, y^{2}\right]$, any irreducible relation $\left[A^{\prime}, B^{\prime}, C^{\prime}\right]$ will be equal to $\left[A_{1}^{\prime} y,-A_{1}^{\prime} x-C_{1}^{\prime} y, C_{1}^{\prime} x\right]$ for some coprime polynomials $A_{1}^{\prime}$ and $C_{1}^{\prime}$ of the same degree $d$.

In this case, $I(A, B, C)=3$ and $I\left(A^{\prime}, B^{\prime \prime}, C\right)=(d+1)^{2}$, whereas the right-hand side of the relation (2) is equal to $(d+3)^{2}-6(d+3)+12=d^{2}+3$. These two numbers cannot agree.

Remark 2. It would also be very interesting to check the following converse of the Darboux lemma: if the Darboux relation holds for some orthogonal system of polynomials, is it necessary that this system be without projective zero?

This natural question is not simple, and the answer will probably be the subject of another paper. Let us simply say that no point $P$ can be a local complete intersection of both ideals $(A, B, C)$ and $\left(A^{\prime}, B^{\prime}, C^{\prime}\right)$ under the assumption that $I\left(A^{\prime}, B^{\prime}, C^{\prime}\right)=I(\ell)$.

\section{Applications}

An affine polynomial vector field $\mathcal{X}=p \partial / \partial x+q \partial / \partial y$ in $\mathbb{C}^{2}$ can be thought of as a $\mathbb{C}$-derivation of the ring $\mathbb{C}[x, y]$, where $p, q \in \mathbb{C}[x, y]$. We say that the degree of $\mathcal{X}$ is the maximum of the degrees of $p$ and $q$.

A projective polynomial vector field $X=P \partial / \partial X+Q \partial / \partial Y+R \partial / \partial Z$ in $\mathbb{P}_{2}(\mathbb{C})$ of degree $d$ is a $\mathbb{C}$-derivation of the ring $\mathbb{C}[X, Y, Z]$, where $P, Q, R \in \mathbb{C}[X, Y, Z]$ are homogeneous polynomials with the same degree $d$. Thus, the triple $[P, Q, R]$ defines a map from the projective plane $\mathbb{P}_{2}(\mathbb{C})$ into itself, with some singularities. We note that, together with the Euler vector field $\mathcal{E}=X \partial / \partial X+Y \partial / \partial Y+Z \partial / \partial Z$, all the $\mathbb{C}$-derivations $\mathcal{X}+A \mathcal{E}$, where $A$ is an arbitrary homogeneous polynomial in $\mathbb{C}[X, Y, Z]$ of degree $d-1$, define the same homogeneous projective Pfaff 1-form of degree $d+1$, providing the same homogeneous foliation of $\mathbb{C}^{3}$ and thus the same foliation of $\mathbb{P}_{2}(\mathbb{C})$; for more details, see $[2,1]$.

If we have an affine polynomial vector field $\mathcal{X}=p \partial / \partial x+q \partial / \partial y$ of degree $d$ in $\mathbb{C}^{2}$, it can be thought of as a projective one, making $p$ and $q$ homogeneous with the same degree $d$ in the variables $X, Y$ and $Z$ as follows:

$$
p \frac{\partial}{\partial x}+q \frac{\partial}{\partial y} \rightarrow Z^{d} p\left(\frac{X}{Z}, \frac{Y}{Z}\right) \frac{\partial}{\partial X}+Z^{d} q\left(\frac{X}{Z}, \frac{Y}{Z}\right) \frac{\partial}{\partial Y},
$$

where we take $R=0$. 
If we have a projective polynomial vector field $\mathcal{X}=P \partial / \partial X+Q \partial / \partial Y+R \partial / \partial Z$ of degree $d$ in $\mathbb{P}_{2}(\mathbb{C})$, it can be thought of as an affine one, considering the vector field $Z \mathcal{X}-R \mathcal{E}$ and taking $Z=1$.

Let $f \in \mathbb{C}[x, y]$. The algebraic curve $f(x, y)=0$ is an invariant algebraic curve of the affine polynomial vector field $\mathcal{X}$ if, for some polynomial $k \in \mathbb{C}[x, y]$, we have

$$
\chi f=\frac{\partial f}{\partial x} p+\frac{\partial f}{\partial y} q=k f .
$$

The polynomial $k$ is called the cofactor of the invariant algebraic curve $f=0$.

It is easy to verify that if $f(x, y)=0$ is an invariant algebraic curve of degree $r$ for the polynomial vector field $\mathcal{X}$ with cofactor $k(x, y)$, then $F(X, Y, Z)=Z^{r} f(X / Z, Y / Z)=0$ is an invariant algebraic curve of degree $r$ for its projective vector field with cofactor $K(X, Y, Z)=Z^{r-1} k(X / Z, Y / Z)$; that is,

$$
\chi F=\frac{\partial F}{\partial X} P+\frac{\partial F}{\partial Y} Q+\frac{\partial F}{\partial Z} R=K F,
$$

where $R=0$.

If $F(X, Y, Z)=0$ is an algebraic curve of $\mathbb{P}_{2}(\mathbb{C})$ of degree $r$, let $p=\left(X_{0}, Y_{0}, Z_{0}\right)$ be a point of $\mathbb{P}_{2}(\mathbb{C})$. Since the three coordinates of $p$ cannot be zero, we can assume without loss of generality that $p=(0,0,1)$. Then suppose that the expression of $F(X, Y, Z)$ restricted to $Z=1$ is

$$
F(X, Y, 1)=F_{i}(X, Y)+F_{i+1}(X, Y)+\ldots+F_{r}(X, Y),
$$

where $0 \leqslant i \leqslant r$, and where $F_{j}(X, Y)$ denotes a homogeneous polynomial of degree $j$ in the variables $X$ and $Y$ for $j=i, \ldots, r$, with $F_{i}$ different from the zero polynomial. We say that $i=m_{p}(F)$ is the multiplicity of the curve $F=0$ at the point $p$. If $i=0$, then the point $p$ does not belong to the curve $F=0$. If $i=1$, we say that $p$ is a simple point for the curve $F=0$. If $i>1$, we say that $p$ is a multiple point.

Proposition 10. Let $f(x, y)=0$ be an irreducible invariant algebraic curve of degree $r \geqslant 1$ without multiple points for the affine polynomial vector field $\mathcal{X}$ of degree $d$. Then $r \leqslant d+1$.

Proof. Since $F=0$ is an invariant algebraic curve of $\mathcal{X}$ with cofactor $K$, we find that

$$
\frac{\partial F}{\partial X} P+\frac{\partial F}{\partial Y} Q=K F
$$

in $\mathbb{P}_{2}(\mathbb{C})$. By using the Euler theorem for the homogeneous function $F$ of degree $r$, this equation becomes

$$
\frac{\partial F}{\partial X}\left(P-\frac{1}{r} X K\right)+\frac{\partial F}{\partial Y}\left(Q-\frac{1}{r} Y K\right)+\frac{\partial F}{\partial Z}\left(-\frac{1}{r} Z K\right)=0 .
$$

Now we take in the Darboux lemma:

$$
A=\frac{\partial F}{\partial X}, \quad B=\frac{\partial F}{\partial Y}, \quad C=\frac{\partial F}{\partial Z}, \quad A^{\prime}=P-\frac{1}{r} X K, \quad B^{\prime}=Q-\frac{1}{r} Y K, \quad C^{\prime}=-\frac{1}{r} Z K,
$$

and

$$
h=I(A \cap B \cap C), \quad h^{\prime}=I\left(A^{\prime} \cap B^{\prime} \cap C^{\prime}\right) .
$$

We note that, by assumption, $h$ and $h^{\prime}$ are finite. Moreover, as $A \cap B \cap C=\emptyset$, we see that $h=0$. 
Since $A, A^{\prime}, B, B^{\prime}, C$ and $C^{\prime}$ satisfy equality (22), the Darboux lemma can be used to obtain

$$
\begin{aligned}
h+h^{\prime} & =\frac{d^{3}+(r-1)^{3}}{d+r-1} \\
& =d^{2}+(r-1)(r-d+1) .
\end{aligned}
$$

By the Bézout theorem, the number of intersection points of the curves $A^{\prime}=0, B^{\prime}=0$ and $C^{\prime}=0$ is at most $d^{2}$, taking into account their multiplicities; that is, $h^{\prime} \leqslant d^{2}$. Hence a lower bound for $h$ is as follows: $0=h \geqslant(r-1)(r-d-1)$, and $1 \leqslant r \leqslant d+1$.

Remark 3. We are not the first to notice the previous result (see, for instance, [1]), but, in our opinion, the Darboux lemma provides a simple and elegant means of obtaining it.

More-involved proofs of this fact can be obtained by taking into consideration the exactness of the Koszul complex built on the vector of partial derivatives of an irreducible homogeneous three-variable polynomial corresponding to a smooth algebraic curve.

On the other hand, Tsygvintsev used the genus-degree formula for a non-singular curve and the Riemann-Hurwitz formula to achieve his proof [5].

In [1], the first two authors have proved the following result, which we shall need later on.

Theorem 11. Let $f(x, y)=0$ be an irreducible algebraic curve of degree $r>1$, which is invariant with cofactor $k \neq 0$, for the affine polynomial vector field $\mathcal{X}$ of degree $d>1$. If $d^{2}$ is the total number of solutions of the system

$$
r P-X K=0, \quad r Q-Y K=0, \quad Z K=0,
$$

in the projective plane, taking their multiplicities or numbers of intersections into account, then $\mathcal{X}$ has a rational first integral.

We recall that a limit cycle of a real affine polynomial vector fields is an isolated periodic orbit in the set of all periodic orbits of the system. An algebraic limit cycle of degree $r$ is an oval of an irreducible invariant algebraic curve $f(x, y)=0$ of degree $r$, which is a limit cycle of the system.

Theorem 12. Real affine quadratic polynomial vector fields have no algebraic limit cycles of degree 3.

Proof. Let $f=0$ be an invariant algebraic curve of degree 3 of a real affine polynomial vector field of degree 2 . If the cubic curve $f=0$ has multiple points, then it is rational (its genus is 0 ) and there is no oval in it. If $f=0$ has no multiple points, equation (23) in the proof of Proposition 10 implies that $h^{\prime}=2^{2}=4$. According to Theorem 11, the system has a rational first integral, and thus no limit cycle.

Acknowledgements. The first two authors are partially supported by DGYCIT grant number PB96-1153 and by CICYT grant number 1999SGR 00349. The third author is very grateful to his university, Paris XII-Val de Marne; the sabbatical semester that he has been granted has greatly facilitated the completion of the present (and other) work. 


\section{References}

1. J. Chavarriga and J. Llibre, 'Invariant algebraic curves and rational first integrals for planar polynomial vector fields', J. Differential Equations 169 (2001) 1-16. 207, 209, 209

2. G. Darboux, 'Mémoire sur les équations différentielles algébriques du premier ordre et du premier degré', Bull. Sci. Math. (2) 2 (1878) 60-96, 123-144, 151-200. 197, 207

3. W. Fulton, Algebraic curves, Math. Lecture Ser. (Benjamin Cummings, 1969); reprinted (Addison-Wesley, 1989). 199, 200

4. J. P. Jounnolou, Equations de Pfaff algébriques, Lect. Notes in Math. 708 (Springer, Berlin, 1979). 198

5. A. Tsygvintsev, Algebraic invariant curves of plane polynomial differential systems', J. Phys. A 34 (2001) 663-672. 209

Javier Chavarriga chava@eup.uds.es

Departament de Matemàtica

Universitat de Lleida

Avda. Jaume II

69. 25001 Lleida

Spain

Jaume Llibre jllibre@mat.uab.es

Departament de Matemàtiques

Universitat Autònoma de Barcelona

08193 Bellaterra

Barcelona

Spain

Jean Moulin Ollagnier Jean.Moulin-Ollagnier@polytechnique.fr

Laboratoire GAGE

UMS CNRS 658 Medicis

École Polytechnique

F 91128 Palaiseau Cedex

France 